\title{
Magnetostriction in $\mathrm{Ar}^{+}$Implanted And Annealed Co/Pd Multilayers
}

\author{
A. D. C. Viegas, L. F. Schelp and J. E. Schmidt \\ Instituto de Física, Universidade Federal do Rio Grande do Sul, CP 15051, 91507-970. \\ Porto Alegre, RS, Brazil
}

\begin{abstract}
Magnetostriction curves with (111) textured polycrystalline $\mathrm{Co} / \mathbf{P d}$ multilayers, as deposited or submitted to $\mathrm{Ar}^{+}$ion implantation or thermal annealing, are presented and discussed. Both treatments, while promoting intermixing among $\mathrm{Co}$ and Pd atoms, induce an increase in the saturation magnetostriction values. The evolution of the engineering curves, however, are different due to different structural modifications promoted by the two treatments.
\end{abstract}

\section{INTRODUCTION}

Among other magnetic properties of these systems, in the last decade some efforts have been made to achieve a better knowledge about the magnetostriction of magnetic multilayers (MLs). The first studies were performed in order to minimize its effects in soft head core materials, like iron/amorphous iron alloy multilayers $[1,2]$. From another point of view, measurements of some annealed $\mathrm{FeCo} / \mathrm{Ag}$ multilayers lead to magnetostriction values greater than that expected for FeCo alloys with the same overall composition [3]. This result suggests that layering can be interesting also to increase the magnetostriction in soft materials, what could be useful for some applications in actuators.

In magnetic MLs, the interfaces can contribute to the overall saturation magnetostriction with a "surface term" [4] (when the interfaces are sharp) or with a contribution emerging from smother chemical modulation through them [5] (when apreciable interface mixing is present). Sometimes one has to take into account the crystallographic texture of the MLs, that may lead to different magnetostriction values compared to those measured in the corresponding polycrystalline bulk materials.

Recently, we have used the well known transition of the magnetic easy axis from an in plane to a perpendicular orientation in $\mathrm{Co} / \mathrm{Pd}$ multilayers [6], to study the effects of different magnetic orientation to the magnetostriction curves [7]. The results have shown that in (111) textured $\mathrm{Co} / \mathrm{Pd}$ MLs the engineering $\left(\lambda_{I, t}\right)$ and saturation $\left(\lambda_{s}\right)$ magnetostrictions are strongly dependent on the Co layer thickness. In multilayers with very thin Co layers $(<\sim 1 n m)$, the $\lambda_{l}$ and $\lambda_{t}$ are large $\left(\sim 10^{-5}\right)$ and negative.

Manuscript received February 15, 1995.

This work was supported by the Conselho Nacional de Desenvolvimento Científico e Tecnológico (CNPq-Brasil), Financiadora de Estudos e Projetos (FINEP-Brasil) and Fundação de Amparo à Pesquisa do Estado do Rio Grande do Sul (FAPERGS).
For thicker Co layers $(\sim 2 n m)$, the magnetic moments tilt toward the sample's plane, and the transversal and longitudinal magnetostrictions decrease one order of magnitude and have opposite signs.

Here we present the results for samples with the same Co thickness, but where different intermixing was achieved by proper annealing or ion implantation. In $\mathrm{Co} / \mathrm{Pd}$, it has been established that these treatments can reduce drastically the effective anisotropy $\left(K_{e f f}\right)$ by the gradual mixing at the interfaces [8]. Concerning the magnetostriction, interesting behavior is observed when one induces simultaneos modifications by creation of an intermixed region at the interfaces and by the change in the magnetic domain orientations.

\section{EXPERIMENTAL}

The Co/Pd multilayers were deposited onto glass and Si substrates by thermal evaporation with rates about 0.1 $\mathrm{nm} / \mathrm{s}$. The measurements in this work refer to samples with thicknesses around 1.0/4.5 nm for the Co and Pd layers thicknesses, respectively. These thicknesses were chosen because they present $K_{\text {eff } f}$ near zero (in our MLs the transition from negative to positive values of $K_{\text {eff }}$ occurs for MLs with $0.7 \mathrm{~nm}$ thickness for the Co layers) and, as a consequence, present magnetic moments with some component out of the plane. After the deposition, the structure of the MLs was gradually changed by submitting the samples to $230 \mathrm{keV} \mathrm{Ar}^{+}$bombardment at room temperature or to annealing in an He atmosphere. The doses used were between $1 \times 10^{13}$ and $1 \times 10^{15}$ ions $/ \mathrm{cm}^{2}$, and the annealing temperatures were up to $450^{\circ} \mathrm{C}$, for 30 minutes each. The hysteresis curves and the effective anisotropy were measured at room temperature using, respectively, a vibration sample and a torque magnetometers. The magnetostriction curves were extracted using the cantilever method proposed by Klockholm[9], with the external field applied parallel to the substrate.

\section{RESUlts AND Discussion}

At our experimental conditions, the structure of the MLs change differently under the two treatments as indicated by the X-ray diffractograms (see Fig.1). In the implanted samples [10], the decrease in the satellites intensities reflects an intermixing process mainly through the interfaces. Intermixing by grain boundaries seems to be important in the annealed samples as indicated by the peak corresponding to $\mathrm{Co}-\mathrm{Pd}$ alloys with different concentrations. 


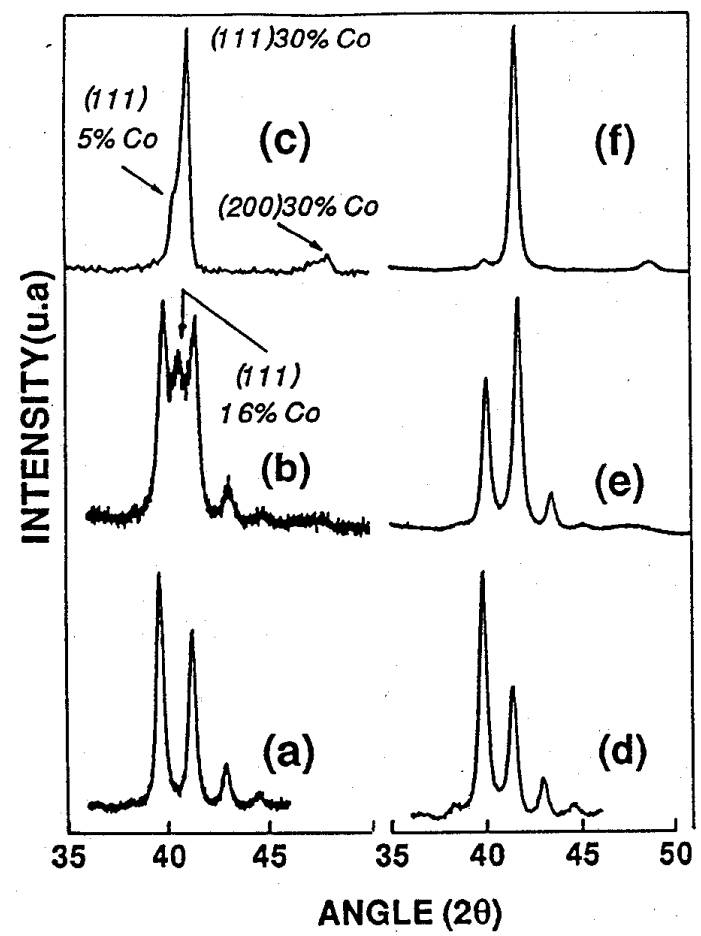

Fig. 1.High angle X-Ray diffraction patterns of the annealed $\mathrm{Co} / \mathrm{Pd}$ multilayers (left) and for the implanted ones (right). The temperatures are (b) $300^{\circ} \mathrm{C}$ and (c) $420^{\circ} \mathrm{C}$. The doses are (e) $1 \times 10^{14}$ and (f) $1 \times 10^{15}$ ions $/ \mathrm{cm}^{2}$. The (a) and (d) patterns refer to the as made samples.

The structural changes affect magnetic properties of the samples. The saturation magnetization $\left(M_{s}\right)$ increases with the dose in the implanted samples and a strong decrease of $K_{\text {eff }}$ occurs even for the lower dose in accordance with prior results [5]. Although we did not detect any oxidation in our annealed samples, the decrease of $M_{s}$ and an almost unchanged $K_{\text {eff }}$ resembles the trends observed in $\mathrm{Co} / \mathrm{Pd} \mathrm{MLs}$ annealed in air, where a columnar structure of the ML is reinforced [6].

The longitudinal and transversal magnetostrictions can be found in the Fig. 2. The as made samples present $\lambda_{l}$ and $\lambda_{t}$ curves with negative signs. This is related to the negative values of the magnetostriction coefficients $\lambda_{A}$ and $\lambda_{B}$ of bulk Co h.c.p ( $\approx-50$ and $-100 \times 10^{-6}$ respectively), and to the fact that the magnetic moments present some component out of the film's plane (without external field), as also observed in some annealed Co films [11].

For the implanted samples (right side of Fig. 2) we can see that the mixing at the interfaces, even for the very low dose used, induces a change in the sign of the $\lambda_{t}$, and an increase of the $\lambda_{s}$ value (as defined in the Fig. 2). The result shows that in $\mathrm{Co} / \mathrm{Pd}$ samples the intermixing alters the magnetostriction in two different ways; i) $\lambda_{s}$ increases because the magnetostriction coefficients of Co-Pd alloys

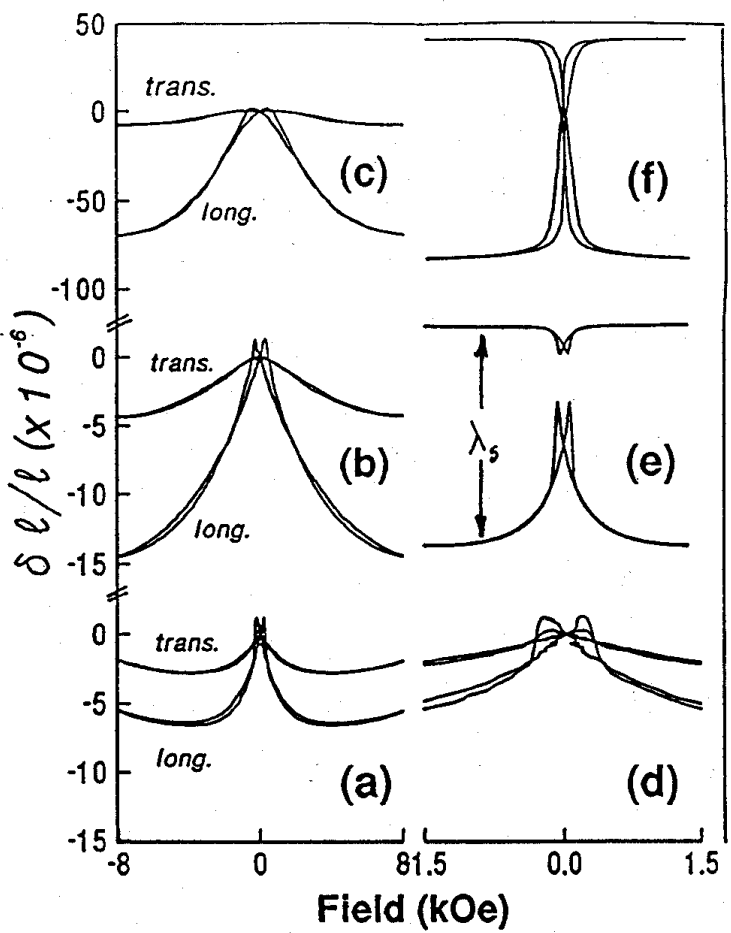

Fig. 2. Longitudinal and transversal magnetostriction curves $\left(\lambda_{l}, \lambda_{t}\right)$ for annealed (left) and implanted $\mathrm{Co} / \mathrm{Pd}$ multilayers (right). a, b, c, d, e and $f$ as defined in Fig. 1. Note the difference in axes scale.

are greater than the Co ones and ii) the engineering curves are sensitive to reorientation of the magnetic moments in the domains as promoted by the decrease in the interface anisotropy value. The decrease observed in the coercive field $\left(H_{c}\right)$ reflects the drop down of $K_{\text {eff }}$ and can also be observed in the magnetization hysteresis loop (not shown).

In the annealed samples, $\lambda_{s}$ also increases by the mixing. among Co and Pd atoms. But, as the columnar morphology of the ML, in this case, is reinforced by the mixing through grain boundaries and the value of $K_{\text {eff }}$ does not decrease, no inversion of the $\lambda_{t}$ curve can be detected. The maintenance of the $K_{\text {eff }}$ value is also responsible for the increase in the field necessary to saturate the sample in the film's plane.

\section{CONCLUSION}

Our results have shown that in Co/Pd multilayers, both annealing and ion implantation induce an increase of the measured values of the saturation magnestostriction. As the Co-Pd alloys magnetostriction coefficients are greater than those of of pure Co, the increase of $\lambda_{s}$ probably reflects the intermixing among Co and $\mathrm{Pd}$ atoms.

The shape and the signs of the engineering magnetostriction curves, however, depend on the unfolding of the interdiffusion process. If the intermixing occurs mainly trough 
the interfaces, this increase is followed by an invertion of the transversal engineering curve that reflect the change in the initial demagnetized state promoted by the drop down in the surface (and effective) anisotropies. This trend can be observed in the samples submitted to $\mathrm{Ar}^{+}$irradiation. If the intermixing occurs by grain boundaries and the columnar structure of the MLs is reinforced, the effective anisotropy do not decrease and no invertion in any of the engineering curves is observed.

\section{REFERENCES}

[1] H. J. de Wit, F. W. A. Dirne and H. M. Witmer, "Magnetic and structural properties of iron/amorphous iron alloy multilayer", J. Appl. Phys., vol. 67, pp. 5131-5133, May 1990.

[2] F. W. A Dirne, J. A. M. Tolboom, H. J. de Wit and C. H. M. Witmer, "Soft Magnetic Multilayers: The role of the magnetostriction", J. Magn. Magn. Mat., vol. 83, pp. 399-401, January 1990.

[3] T. A. Lafford, M: R. J. Gibbs and C. Shearwood, "Magnetic, magnetostrictive and structural properties of ironcobalt/ silver multilayers", J. Magn. Magn. Mat., vol. 132, pp. 89-94, April 1994.

[4] R. Krishnan, "Magnetic multilayers: fundamental and practical aspects", J. de Phys. IV, C3, vol. 2, pp. 159167, December 1992.

[5] F. W. A. Dirne and C. J. M. Denissen, "Interface Mixing in Fe/Co multilayers", J. Magn. Magn. Mat., vol. 78, pp. 123-128, February 1989.

[6] F. J. A. den Broeder and H. C. Donkersloot, "Magnetic properties and structure of $\mathrm{Pd} / \mathrm{Co}$ and $\mathrm{Pd} / \mathrm{Fe}$ multilayers", J. Appl. Phys., vol. 61, pp. 4317-4319, April 1987.

[7] L. F. Schelp, A. D. C. Viegas, M. Carara and J. E. Schmidt, "Magnetostriction of Co/Pd multilayers", J. Magn. Magn. Mat., vol. 139, pp. 59-64, January 1995.

[8] F. J. A. den Broeder, D. Kuiper and H. J. G. Draaisma, "Effects of annealing and ion implantation on the magnetic properties of Pc/Co multilayers containing ultrathin $\mathrm{Co}^{\text {" }}$, IEEE Trans. Magn., vol. 23, pp.3696-3698, September 1987.

[9] E. Klokholm, "The measurement of magnetostriction in ferromagnetic thin films", IEEE Trans. Magn., vol. 12, pp. 819-821, 1976.

[10] L. F. Schelp, M. Carara, A. D. C. Viegas, M. A. Z. Vasconcellos, and J. E. Schmidt, "Structural and magnetic behavior of $\mathrm{Ar}^{+}$-implanted Co/Pd multilayers : interfacial mixing", J. Appl. Phys., vol. 75, pp. 5262-5266, May 1994.

[11] H. Yamane, Y. Maeno and M. Kobayashi, "Annealing effects on multilayered $\mathrm{Co} / \mathrm{Pt}$ and $\mathrm{Co} / \mathrm{Pd}$ sputtering films", Appl. Phys. Lett., vol. 62, pp. 1562-1564, 1993. 